\title{
Déprescrire en EMS: regards croisés entre les résidents, leurs proches et les professionnels de la santé
}

\author{
$\overline{\text { DAMIEN CATEAU }}{ }^{\mathrm{a}, \mathrm{b}, \mathrm{c}}$, ROSE-ANNA FOLEY $^{\mathrm{d}, \mathrm{e}}$ et ANNE NIQUILLE ${ }^{\mathrm{a}, \mathrm{b}, \mathrm{c}}$
}

Rev Med Suisse 2020; 16: 2169-71

\begin{abstract}
Déprescrire pour réduire l'usage de médicaments inappropriés et, plus généralement, diminuer la polymédication reste un défi, en particulier chez les résidents d'établissements médico-sociaux. Les professionnels de la santé actifs en institution perçoivent cette population comme réticente au changement et peu encline à s'engager dans une telle démarche. Pourtant, les résultats de trois études, les deux premières qualitatives, la dernière quantitative, indiquent que ces résidents, ainsi que leurs proches, seraient prêts à tester une réduction de leur traitement, pour autant que l'on prenne le temps de discuter avec eux des bénéfices potentiels.
\end{abstract}

\section{Deprescribing in nursing homes: comparative views of residents, their relatives, and healthcare professionals}

Deprescribing, in order to reduce both polypharmacy and the use of potentially inappropriate medications, remains a challenge, especially in nursing homes. Healthcare professionals perceive residents of these homes as wary of change and reluctant to take part in such endeavours. The results of two studies, one qualitative and the other quantitative, show that, on the contrary, nursing home residents and their relatives would be ready to consider a treatment reduction, provided that time is invested to explain the expected benefits of such changes.

\section{INTRODUCTION}

«Déprescrire»: un verbe encore absent des dictionnaires de langue française, mais qui prend tout son sens en gériatrie. Conçue comme une réponse à la polymédication et à l'usage de médicaments inappropriés, la déprescription s'inscrit dans la continuité de la concertation soignant-patient autour du traitement. Malgré des résultats prometteurs issus de plusieurs essais cliniques, mettre en œuvre une intervention de déprescription reste délicat, particulièrement en établissement médico-social (EMS). Qu'en pensent les résidents, leurs proches et les professionnels de la santé qui les entourent?

aPharmacie d’Unisanté, Centre universitaire de médecine générale et santé publique, Université de Lausanne, 1011 Lausanne, ${ }^{b}$ Section des sciences pharmaceutiques, Université de Genève, 1211 Genève 4, ' Institut des sciences pharmaceutiques de Suisse occidentale, Université de Genève, 1211 Genève 4 et Université de Lausanne, 1011 Lausanne, 'dSecteur Systèmes et services de santé, Centre universitaire de médecine générale et santé publique, Université de Lausanne, 1011 Lausanne, 'Unité de recherche en santé, Haute École de santé Vaud, 1011 Lausanne

damien.cateau@unisante.ch | rose-anna.foley@unisante.ch anne.niquille@unisante.ch
Déprescrire consiste à retirer les traitements jugés néfastes ou inappropriés, ou à réduire leur dose, dans le but d'atténuer les effets de la polymédication et d'améliorer les résultats de santé. ${ }^{1}$ La déprescription diffère d'un simple arrêt ou d'une non-adhésion thérapeutique par sa systématicité (c'est-à-dire, recherche active de traitements à problème): la supervision par un professionnel de la santé, la concertation avec le patient, et l'objectif d'améliorer la situation de ce dernier. L'utilité de la déprescription commence à être bien établie: une revue systématique parue en 2018, focalisée sur les interventions de déprescription chez les résidents d'EMS, a montré une diminution significative de l'usage de médicaments inappropriés, de l'ordre de 60\% (odds ratio (OR): 0,41; intervalle de confiance (IC) à $95 \%$ : $0,19-0,89) .^{2}$ De plus, une réduction significative de la mortalité (réduction de 26\%; OR: 0,74; IC 95\%: $0,65-0,84)$ et une baisse de $24 \%$ du nombre de patients ayant chuté au moins une fois (OR: 0,76; IC 95\%: 0,62-0,93) ont été constatées dans les études qui ont testé des interventions de type «revue de médication focalisée sur la déprescription». Une revue systématique antérieure, qui a analysé les études menées dans la population gériatrique générale, a également souligné un bénéfice en termes de mortalité pour ce type d'interventions (OR: 0,62; IC 95\%: 0,43-0,88). ${ }^{3}$

Ces résultats sont encourageants, mais la prise de médicaments reste ancrée dans un réseau complexe d'attitudes et de convictions, tant du côté des soignants que des patients et de leurs proches. De nombreuses barrières à la déprescription ont ainsi été mises en évidence dans la littérature. La peur des conséquences d'un arrêt, la croyance en un bénéfice futur d'un traitement, ou encore l'insécurité d'un prescripteur quant à la manière de mener un arrêt sont quelques-uns des nombreux freins à la mise en œuvre de la déprescription. ${ }^{4}$

Afin d'adapter deux interventions de déprescription dans des EMS vaudois et fribourgeois à la réalité du terrain, nous avons cherché à comprendre la perception que tous les acteurs impliqués, y compris les résidents et leurs familles, ont de la déprescription. Nous avons donc mené deux études qualitatives, l'une focalisée sur les résidents et leurs proches, l'autre sur les professionnels de la santé exerçant en EMS (médecins, pharmaciens et infirmières). Elles ont été complétées par une troisième étude, celle-ci quantitative, liée à la validation de la traduction française du questionnaire «Revised Patients' Attitude Towards Deprescribing» (Attitude des personnes âgées vis-à-vis de la déprescription (APAD)). ${ }^{5}$ Nous proposons ici une synthèse croisée des résultats de ces études, qui ont fait l'objet de publications séparées. ${ }^{6,7}$ 


\section{RÉSIDENTS GLOBALEMENT PRÊTS FACE À LA DÉPRESCRIPTION}

La première étude, consacrée aux résidents et à leurs proches, s'est déroulée dans quatre EMS, répartis entre les cantons de Vaud et Fribourg; douze entretiens y ont été réalisés entre 2017 et 2018 par une sociologue et une anthropologue de la santé. Onze entretiens ont impliqué des résidents, dont trois accompagnés d'un proche, et un entretien a été réalisé uniquement avec la fille d'un résident. Les thématiques majeures identifiées lors de l'analyse de ces entretiens figurent dans le tableau 1.

Leur perception de la déprescription, et plus globalement de la problématique de la polymédication, est similaire à celle décrite dans la littérature ${ }^{4}$ et dénote une grande ambivalence. De nombreux résidents et proches ont exprimé une lassitude importante face à la consommation de médicaments ( «J'en prends tellement!», «Je trouve que j'en ai trop des médicaments.»), ${ }^{a}$ et aimeraient pouvoir s'en passer autant que possible («Je souhaite qu'on ne consomme aucun médicament si c'est possible!»), tout en manifestant une grande confiance dans les traitements qu'ils jugent bénéfiques: «Les gouttes dans les yeux, je pense que ça, c'est indispensable. Ça va pas me guérir mais disons que ça empêche peut-être au glaucome de progresser $[. .]$.$» , «Pour le moment ça va bien, je touche du bois! Tant que ça$ va bien, je vais pas arrêter [le médicament pour l'estomac]». Aucune des personnes interrogées ne semble préoccupée par les questions d'interactions médicamenteuses ou d'effets indésirables à long terme, associant d'éventuels symptômes négatifs à la progression d'une pathologie plutôt qu'à un traitement.

Concernant leur traitement, les participants à cette étude s'en remettent fortement aux décisions du prescripteur; ceci a été confirmé par les résultats de la validation du questionnaire APAD: si seuls $45 \%$ des 73 résidents interrogés seraient tentés d'arrêter un traitement de leur propre chef pour voir comment ils se sentent sans celui-ci, la totalité le ferait sur conseil de leur médecin. L'immense majorité (96\%) de ces résidents déclarent qu'ils ne considéreraient pas cela comme un abandon thérapeutique, alors que leurs proches (46 personnes interrogées) se sont montrés plus partagés:35\% d'entre eux déclarent qu'ils percevraient une proposition d'arrêt comme un renoncement à soigner. Bien entendu, l'avis favorable des résidents ne garantit pas l'absence de réticences une fois la question pratique de l'arrêt posée.

Une thématique importante présente dans les entretiens est le fort sentiment de dépossession ressenti par les résidents d'EMS, dans de nombreux aspects de leur vie quotidienne ( $\ll$ Moi je voudrais pouvoir faire ma toilette et puis après sortir quand j'en ai envie! [...] Pas qu'on me dise "Aujourd'hui vous irez à telle et telle place"»), ainsi que dans leur prise en charge thérapeutique: «L'infirmière elle vient et puis elle me dit: "Mme K. aujourd'hui faut prendre ce médicament." [...] Mais pourquoi je prends un médicament? "C'est parce que vous avez du mal à penser normalement.” (Certains proches de résidents ressentent des manquements dans la communication

aLes citations ont été condensées dans cet article; les références exactes avec noms d'emprunt, sexe et âge sont détaillées dans les publications originales. ${ }^{6,7}$

\begin{tabular}{|c|c|c|c|c|}
\hline & TABLEAU I & \multicolumn{2}{|c|}{$\begin{array}{l}\text { Déterminants de } \\
\text { la déprescription en EMS }\end{array}$} & \\
\hline \multicolumn{5}{|c|}{ Point de vue des résidents et de leurs proches. } \\
\hline \multicolumn{3}{|c|}{$\begin{array}{l}\text { Efficacité ressentie vs risques } \\
\text { potentiels }\end{array}$} & \multicolumn{2}{|c|}{$\begin{array}{l}\text { Institution structurante dans la } \\
\text { consommation de médicaments }\end{array}$} \\
\hline \multicolumn{3}{|c|}{$\begin{array}{l}\text { - Souhait de n'utiliser que les médica- } \\
\text { ments nécessaires } \\
\text { - Inconfort de la prise } \\
\text { - Perception de l'(in)efficacité } \\
\text { - Perception des effets indésirables } \\
\text { - Confiance à l'égard du prescripteur } \\
\text { - Gestion du risque médicamenteux } \\
\text { déléguée aux professionnels mais sen- } \\
\text { sibilisation aux risques de la polymédi- } \\
\text { cation }\end{array}$} & \multicolumn{2}{|c|}{$\begin{array}{l}\text { - Suivi médical via le personnel infir- } \\
\text { mier } \\
\text { - Méconnaissance des médicaments } \\
\text { - Observance forcée consentie } \\
\text { - Sentiment de dépossession de soi } \\
\text { - Éloignement des proches quant } \\
\text { aux décisions sur les médicaments }\end{array}$} \\
\hline
\end{tabular}

avec les soignants («Une fois ils avaient rajouté de la morphine. Ça avait des effets secondaires de nausées [...] ça, elles m’en avaient pas parlé les infirmières [...]»), sans toutefois revendiquer systématiquement une plus grande implication dans les décisions liées au traitement du résident qu'ils accompagnent.

\section{MÉDECINS ET SOIGNANTS PROJETANT PARFOIS DES CRAINTES INFONDÉES}

La deuxième étude a été réalisée auprès des professionnels de la santé exerçant en EMS. Deux focus-groups ont réuni d'une part onze pharmaciennes et pharmaciens, et d'autre part dix infirmières et infirmiers; le troisième focus-group prévu pour les médecins n'a pas pu avoir lieu pour des raisons organisationnelles. Il a été remplacé par six entretiens individuels. Le tableau 2 résume les facteurs clés identifiés lors de cette étude, qui interviennent dans la démarche de déprescription en EMS; la majorité d'entre eux peut aussi bien faciliter la démarche que lui faire obstacle, selon les spécificités de la situation.

Les trois groupes de professionnels perçoivent les résidents d'EMS comme réticents à reconsidérer un traitement, que ce soit à cause d'un bénéfice direct perçu, comme pour les somnifères ou les laxatifs («Celui pour dormir ils n’oublient pas et puis les poudres, les sachets ça voilà c'est aussi un peu leur raison de vivre d'aller aux toilettes tous les jours»), ou en raison d'une addiction, d'un attachement à un prescripteur historique («On [est] quand même face à des personnes qui sont encore dans “oui mais moi c'était mon Dr Untel qui m’a donné ça et puis je vais continuer à le prendre”»), ou encore d'une incapacité au changement liée à l'âge: «C'est pas à 90 ans qu'ils vont reconcevoir les choses.» Les familles des résidents sont identifiées comme très protectrices, surtout en cas de troubles cognitifs, ce qui les conduit à s'opposer parfois à la déprescription («il y [a] des familles qui disent "mais ma maman a besoin de ça parce que vous savez elle a fait une dépression [...], faut pas lui enlever l'antidépresseur, il faut pas lui enlever le tranquillisant du soir sinon elle dort pas"»), tout en étant demandeuses d'une maîtrise de la consommation médicamenteuse: «la grande majorité quand même demande à ne pas trop donner de médicaments».

Médecins, infirmières et pharmaciens s'accordent sur le fait que proposer l'arrêt d'un traitement pourrait être perçu comme un désintérêt thérapeutique; au vu des réponses au 


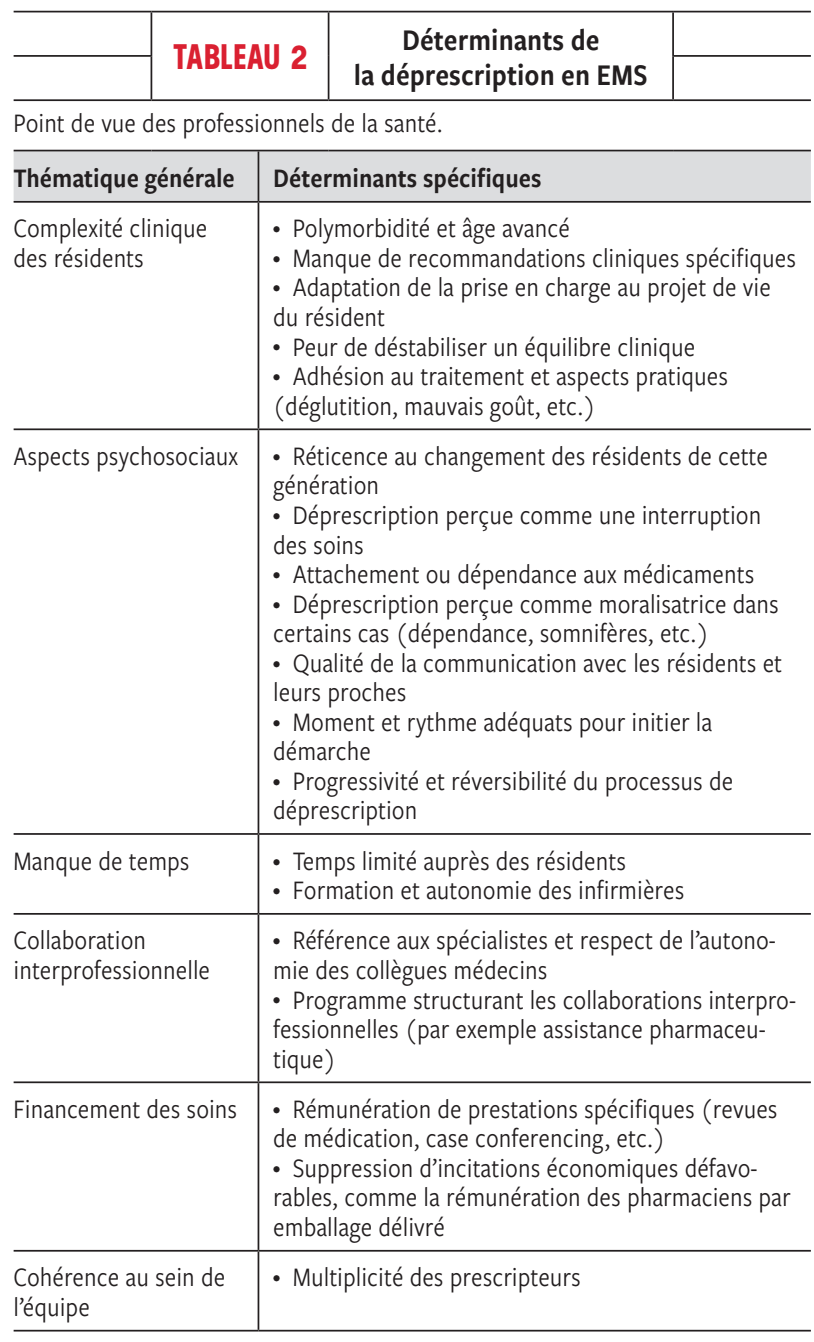

questionnaire APAD, cela semble être une préoccupation pour les proches, mais pas pour les résidents.

Les professionnels relèvent que de nombreux aspects organisationnels font obstacle à la mise en œuvre d'interventions de déprescription: le manque de temps à disposition avec les résidents et leurs familles, la difficulté à implémenter des approches non médicamenteuses par manque de personnel formé, ou encore la multiplicité des acteurs intervenant en EMS. Ces points sont étroitement liés au cadre financier de l'activité en EMS, ainsi qu'à l'absence de rémunération spécifique pour des activités de type revue de médication, qu'elles soient réalisées par le médecin, le pharmacien, ou en équipe.

\section{CONCLUSION}

Les barrières organisationnelles à l'introduction de la déprescription en EMS identifiées par les professionnels ne sont pas nouvelles, et sont en grande partie inhérentes à l'organisation du système de santé suisse. Une importante barrière envisagée par ces mêmes professionnels, la supposée réticence des résidents, semble par contre surmontable. En effet, ces derniers ont manifesté un a priori positif par rapport à la déprescription dans l'étude qualitative et au travers du questionnaire APAD. Le principal frein semble plutôt être la confiance que les résidents et leur famille accordent aux médicaments prescrits de longue date et dont ils ne remettent pas en question la balance bénéfice-risque au fil de l'évolution de leur état de santé. Il semble donc nécessaire de créer des espaces de communication permettant de discuter des changements de traitement, et surtout des bénéfices attendus, avec les résidents et leurs proches. Cela répondrait aux inquiétudes quant au renoncement à soigner exprimées par les proches et à la perte de contrôle sur leur prise en charge ressentie par de nombreux résidents.

Plusieurs projets visant à optimiser la prise en charge médicamenteuse des résidents d'EMS sont actuellement en cours en Suisse. L'un d'entre eux, ${ }^{8}$ mené par certains des auteurs de cet article et dont les résultats sont en cours d'analyse, a intégré dans sa méthodologie un tel espace de dialogue. L'analyse des questionnaires de satisfaction remplis par les participants et leurs proches, ainsi que les entretiens qualitatifs menés avec les professionnels impliqués, permettront de dire si cette approche améliore la communication entre professionnels, résidents et proches.

Conflit d'intérêts: Les auteurs n'ont déclaré aucun conflit d'intérêts en relation avec cet article.

\section{IMPLICATIONS PRATIQUES}

- Les résidents d’établissements médico-sociaux, ainsi que leurs proches, sont prêts à tenter la déprescription de certains traitements, pour autant que leurs médecins le leur proposent

- La mise en œuvre de changements de traitement nécessite de ménager des espaces de discussion

- Travailler en équipe interprofessionnelle permettrait de surmonter les barrières organisationnelles qui restent importantes
1 *Reeve E, Gnjidic D, Long J, Hilmer S. A systematic review of the emerging definition of « deprescribing » with network analysis: Implications for future research and clinical practice. Br J Clin Pharmacol 2015;80:1254-68.

2 **Kua CH, Mak VSL, Huey Lee SW. Health Outcomes of Deprescribing Interventions Among Older Residents in Nursing Homes: A Systematic Review and Meta-analysis. J Am Med Dir Assoc 2018;20:362-72.

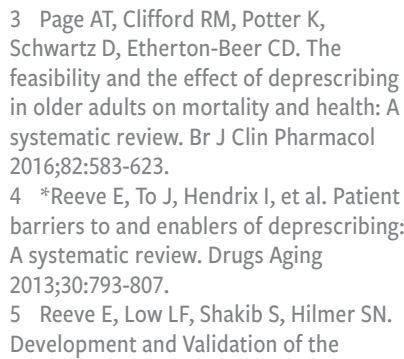

Revised Patients' Attitudes Towards Deprescribing (rPATD) Questionnaire: Versions for Older Adults and Caregivers. Drugs Aging 2016;33:913-28. 6 Foley R-A, Hurard LL, Cateau D, et al. Physicians', Nurses' and Pharmacists' Perceptions of Determinants to Deprescribing in Nursing Homes Considering Three Levels of Action: A Qualitative Study. Pharmacy (Basel) 2020;8.

7 Lechevalier Hurard L, Cateau D,

Bugnon O, Niquille A, Foley R-A. Points de vue d'usagers sur la déprescription de médicaments en maison de retraite. Gerontol Soc 2020;42:171-87.

8 Cateau D, Balabeni P, Mena S, Bugnon O, Niquille A. Deprescribing in nursing homes: Protocol for nested, randomised controlled hybrid trials of deprescribing interventions. Res Socia Adm Pharm 2020 ;epub ahead of print.

* à lire

** à lire absolument 